\title{
Growth of Aspergillus nidulans in a Thin Liquid Layer
}

\author{
By B. L. COHEN \\ Department of Genetics, University of Glasgow, Glasgow, G I I $5 J S$
}

(Received I3 November 1972; revised 21 December 1972)

SUMMAR Y

Growth of Aspergillus nidulans in a thin layer of liquid minimal medium was exponential. The dry-weight doubling time at $37^{\circ} \mathrm{C}$ was $\mathrm{I} \cdot \mathrm{O} \mathrm{h}$ when the inoculum density was $1.3 \times 10^{5}$ viable spores $/ \mathrm{ml}$. Compared with shake-flask cultures, growth was equally fast, but exponential growth ceased sooner. Aerial conidiation occurred, however, and the method was simple and permitted the collection of extracellular products in the absence of cell breakage.

\section{INTRODUCTION}

It is common practice, when growing filamentous fungi such as Aspergillus nidulans for biochemical analysis, to use stationary liquid surface cultures or, more commonly, liquid submerged cultures in shake flasks or stirred fermenters. Surface cultures show normal morphological development and conidiate normally but are difficult to inoculate reproducibly, whereas submerged cultures are reproducible but often do not conidiate well.

In genetic analysis, growth of colonies is normally upon agar-solidified media and morphological development proceeds in a way more comparable with surface than with submerged cultures. Moreover, biochemical changes associated with maturation and conidiation may be anticipated and have been observed by, for example, Clutterbuck (1972). In the course of a genetic and biochemical analysis of extracellular enzyme production by Aspergillus nidulans a method of culture was sought that would permit the harvesting of mycelium of different ages together with the collection of extracellular enzymes and yet would permit growth in a form reasonably similar to that shown by a colony on solid medium. The method of Clutterbuck (1972) in which spores are spread in a thin liquid layer over nutrient medium in Petri dishes was therefore modified by the omission of the agar. It was found that growth kinetics in such cultures were very favourable.

\section{METHODS}

General techniques, media and strains. General techniques and media were those described by Pontecorvo, Roper, Hemmons, MacDonald \& Bufton (1953). Minimal medium was modified by the omission of sodium nitrate and the addition of ammonium tartrate ( 50 mm-ammonium) as nitrogen source. The strain used was $b i A r$, a biotin-requiring derivative of the Glasgow wild-type; it was strain number 05I from the Glasgow collection. Minimal medium was supplemented with biotin $(0.4 \mu \mathrm{g} / \mathrm{ml})$.

Preparation of spore suspensions. Conidia were harvested from complete medium slopes, solidified with $3 \%$ (w/v) agar, by scraping with a wire loop and suspending in Tween 80 $(0.01 \%)$. After vigorous agitation the spores were washed twice by centrifuging and resuspending in water. Slopes up to I month old were used. Conidia were counted, after suit- 


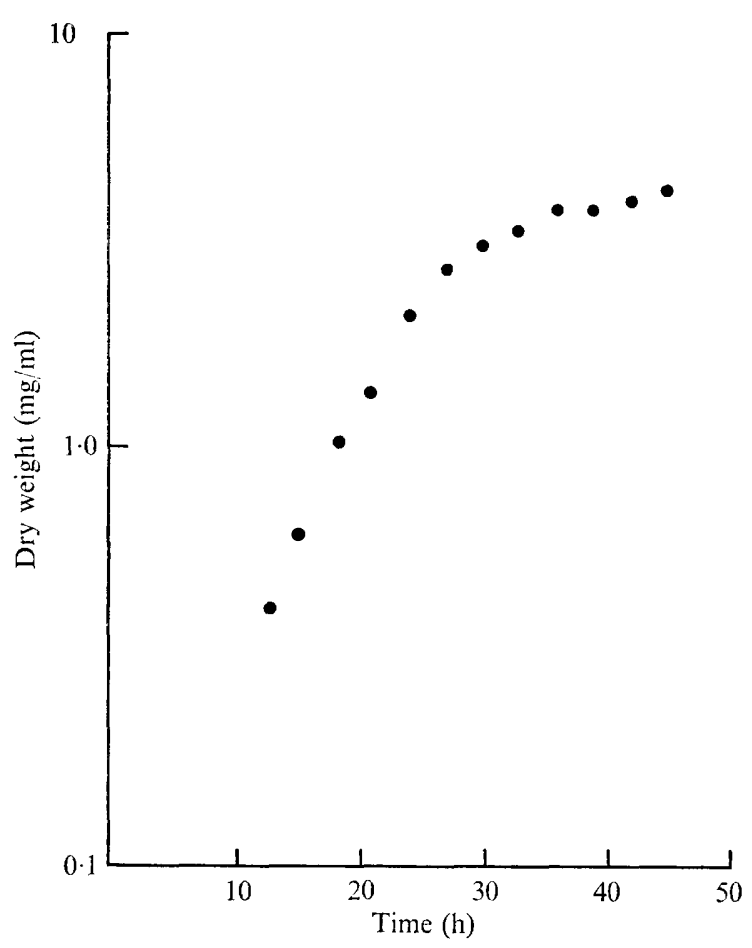

Fig. I. Growth of Aspergillus nidulans biAI on ammonium-minimal medium plus biotin; $10^{6}$ spores $/ \mathrm{ml}$ in Petri dish liquid-layer culture.

able dilution, in a haemocytometer chamber and viable counts were obtained by plating out on biotin-supplemented minimal medium.

Comparison of shake-flask and Petri dish liquid-layer cultures. Medium was inoculated with washed spores and distributed either in Io $\mathrm{ml}$ lots into triple-vented polystyrene Petri dishes (90 mm diam., Sterilin Ltd, Hill Rise, Richmond, Surrey) or in $25 \mathrm{ml}$ lots into triply dimpled $250 \mathrm{ml}$ conical flasks with cotton-wool plugs. Petri dishes were incubated at $37^{\circ} \mathrm{C}$ on a levelled shelf. Conical flasks were incubated at $37^{\circ} \mathrm{C}$ in an orbital shaking incubator, 200 cycles/min. Since $10 \mathrm{ml}$ of minimal medium did not spread readily over the whole area of a $90 \mathrm{~mm}$ Petri dish, Tween 80 (0.0001 \% final concentration) was added to the medium. This was not enough to cause troublesome foaming in the shake flasks.

The contents of culture vessels were harvested by suction filtration in tared, sintered-glass crucibles (GallenkampiLtd, porosity X2), washed with water and dried overnight at $105{ }^{\circ} \mathrm{C}$. Tare and gross weights were obtained after cooling over silica-gel desiccant. For each culture sample three Petri dishes $(30 \mathrm{ml})$ or two shake flasks $(50 \mathrm{ml})$ were harvested and their contents pooled.

\section{RESULTS}

The kinetics of growth of Aspergillus nidulans biAI on minimal medium in Petri dish liquid-layer culture are shown in Fig. I. There was a period of exponential growth, terminating about $25 \mathrm{~h}$ after inoculation, followed by a gradual transition into a slower growth phase. The reproducibility of the method is demonstrated in Fig. 2 where part of the exponential growth phase of four independent replicate cultures is represented. In each case the 


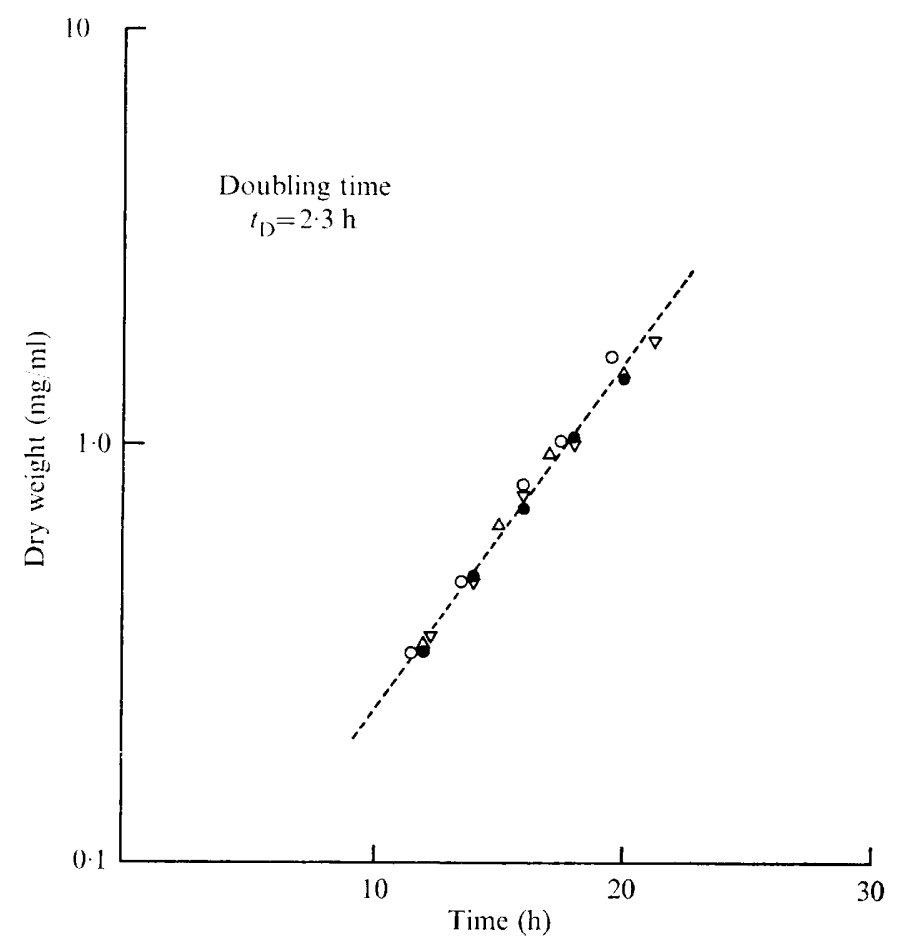

Fig. 2. Growth of Aspergillus nidulans biAr on ammonium-minimal medium plus biotin; $10^{6}$ spores $/ \mathrm{ml}$ in Petri dish liquid-layer culture. Results of four independent replicate experiments.

inoculum density was $10^{6} / \mathrm{ml}$ as determined by haemocytometer counts. A doubling time of $2.3 \mathrm{~h}$ is indicated by the line drawn through these points. Growth rates in liquid layer cultures at inoculum densities ranging from $\mathrm{I} \cdot 3 \times 10^{5}$ to $4.0 \times 10^{6} / \mathrm{ml}$ (viable counts) are shown in Fig. 3. Doubling times range from $\mathrm{I} \cdot 0 \mathrm{~h}$ for the lowest to $3.75 \mathrm{~h}$ for the highest inoculum.

Comparison of growth rates in liquid-layer and in shake-flask cultures may be obtained from Fig. 2 to 4 . With an inoculum density of $1 \cdot 3 \times 10^{5} / \mathrm{ml}$ doubling times are similar $\left(t_{\mathrm{D}}=\mathrm{I} \cdot 0 \mathrm{~h}\right.$ in liquid layer, $\mathrm{I} \cdot 25$ in shake flask) though critical comparison is precluded by the error inherent in line-fitting. With $10^{6}$ spores $/ \mathrm{ml}$, however, the doubling time in liquid layer increased to $2 \cdot 3 \mathrm{~h}$ (Fig. 2) whilst that in shake flasks remained close to I.25 h (Fig. 4).

A major difference between liquid-layer and shake-flask cultures concerns the cell mass at which exponential growth ceased. In liquid layers (Fig. I, 3) this was slightly above $3 \mathrm{mg}$ dry weight $/ \mathrm{ml}$, after which growth continued at a lower, non-exponential rate (Fig. I). In shake flasks, however, exponential growth ceased at about $6 \mathrm{mg}$ dry weight/ml (Trinci, 1969; Cohen, unpublished results). It seems probable that the factor responsible for the early decline of exponential growth in Petri dish cultures was gas exchange and that a cell mass corresponding to about $3 \mathrm{mg}$ dry weight $/ \mathrm{ml}$ represented the greatest quantity of exponentially growing mycelium that could obtain unlimited gas exchange under these particular stationary culture conditions.

In liquid-layer cultures at inoculum densities below about $10^{6} / \mathrm{ml}$ the mycelium formed a loosely interwoven mat, confluent at later times and floating freely just below the surface of the medium. Aerial hyphae protruded towards the surface and conidiophores of normal 


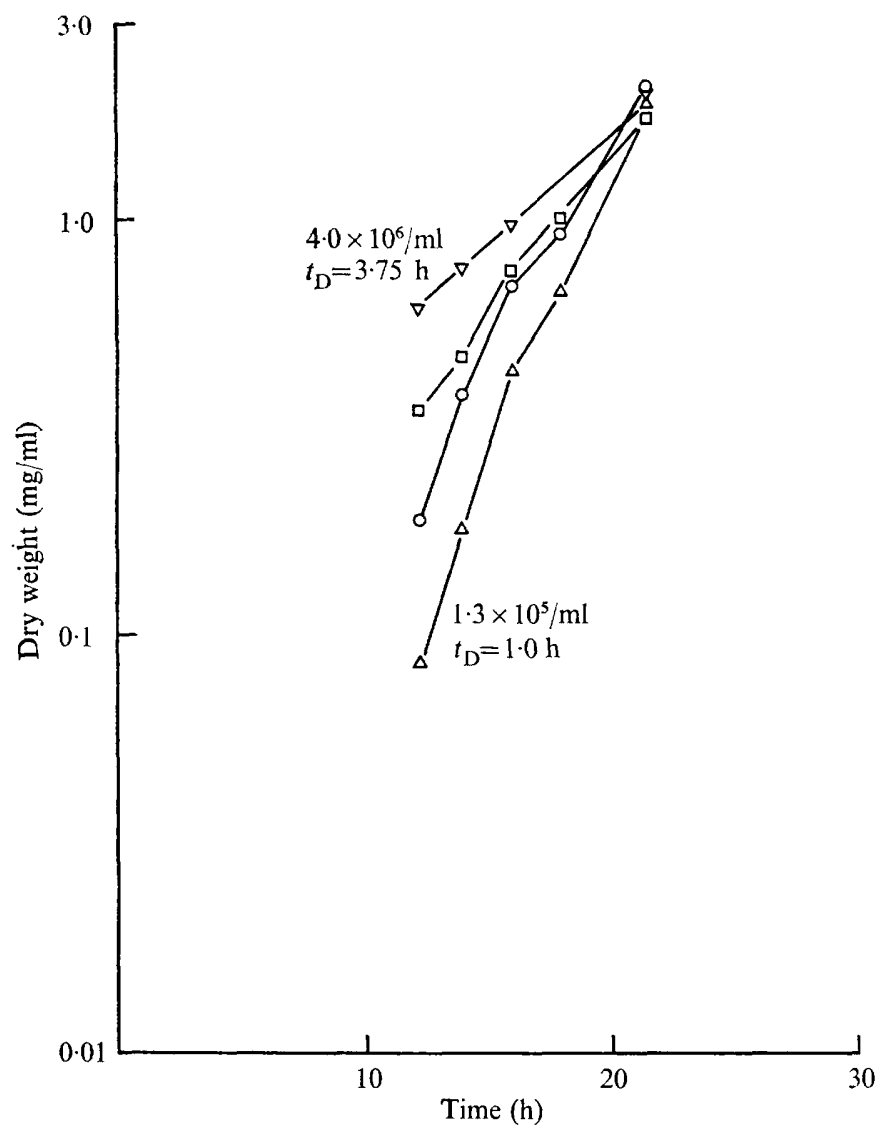

Fig. 3. Growth of Aspergillus nidulans biAt on ammonium-minimal medium plus biotin in Petri dish liquid-layer culture at different inoculum densities. $\triangle, \mathrm{I} \cdot 3 \times 10^{5} / \mathrm{ml} ; 0,3.7 \times 10^{5} / \mathrm{ml}$; $\square, 7.5 \times 10^{5} / \mathrm{ml} ; \nabla, 4.0 \times 10^{6} / \mathrm{ml}$.

morphology were produced. By the time conidiation was fully developed the mat of mycelium entirely filled the liquid layer and its hydrophobic upper layer was exposed to the air. On suitable media conidial maturation proceeded normally.

During that part of the exponential phase when mycelial bulk was appreciable (i.e. 15 to $20 \mathrm{~h}$ after inoculation) the mycelium could readily be harvested by emptying the dishes and filtering on gauze cloth. Such mycelium could be easily redispersed in fresh medium by gentle shaking and this suspension, even if concentrated several-fold, could be uniformly pipetted provided that the pipette had its orifice enlarged by cutting or grinding away the tip.

\section{DISCUSSION}

The culture method described here combines the ease of inoculation and harvesting and the reproducibility of shake flasks with a close approach to the morphological sequence associated with growth on the surface of liquid or solid media. It has several advantages and some limitations. The advantages include freedom from dependence on mechanical agitation, compactness, simplicity, uniformity of replicate cultures, ease of harvesting and fast exponential growth. A particular advantage is the possibility of collecting cellular secretion 


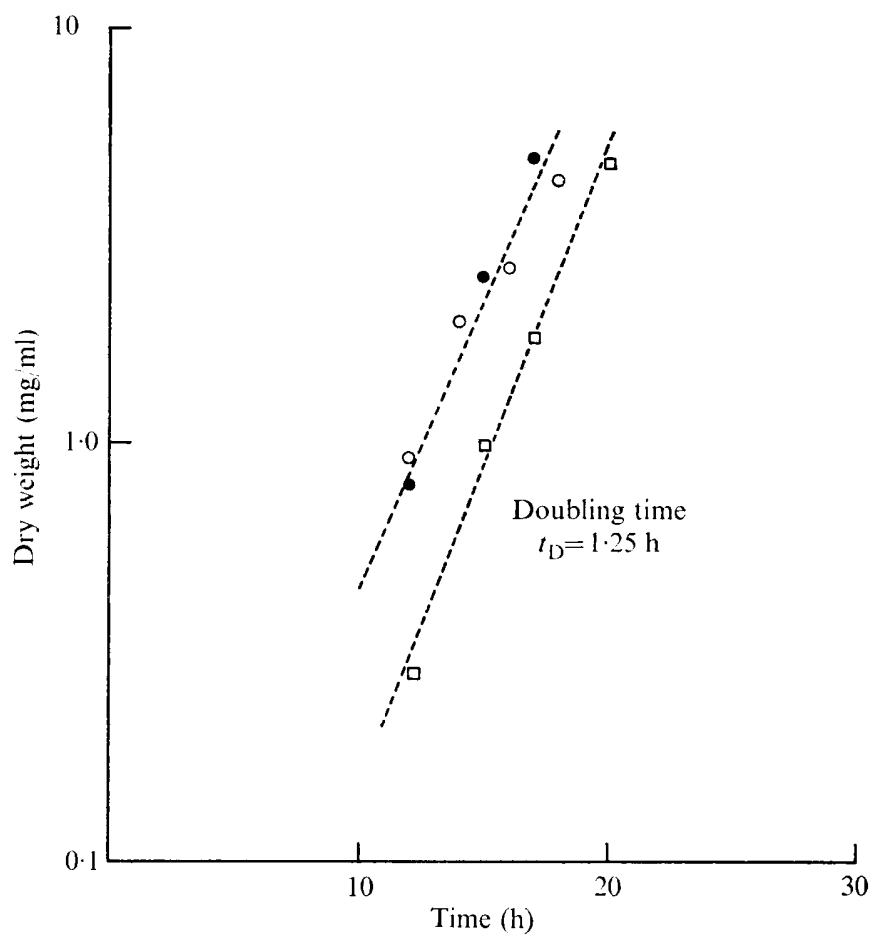

Fig. 4. Growth of Aspergillus nidulans biAI on ammonium-minimal medium plus biotin in shakeflask cultures at different inoculum densities. $\square, 10^{5} / \mathrm{ml} ; O$ and $\bullet, 10^{6} / \mathrm{ml}$. (Replicate experiments.)

products in cultures free from cell rupture caused by agitation and in a state of morphological development similar to that shown by cultures on solid media.

The chief limitation of the liquid-layer method is that the harvestable cell mass is about two-fifths of that which would be obtained from comparable shake-flask cultures.

At high inoculum density the growth rate 12 to $20 \mathrm{~h}$ after inoculation was slower than that obtained in shake flasks (Trinci, 1969) but at lower inoculum density the growth rates were similar. This density-dependent effect on growth rate (Fig. 3) may be a disadvantage of the method and is not at present understood. 'Auto-inhibition' of germination at high densities has been recognized (Bainbridge, I97I) and, consistent with this, I2-h-old Petri dish cultures inoculated at $4.0 \times 10^{6}$ spores $/ \mathrm{ml}$ did contain a large number of germinated spores whose germ-tube extension had stopped. However, if this is the sole circumstance affecting densely inoculated cultures then it is difficult to see why the growth rate of the culture as a whole, i.e. of those germlings that have escaped 'auto-inhibition', is reduced. Alternative explanations might invoke some effect due to oxygen limitation or the accumulation of a growth inhibitor. However, whilst oxygen limitation or gas exchange generally might govern the actual timing of the end of exponential growth in these cultures ${ }_{y}$ it is not obvious that it can account for the differing exponential growth rates of different inocula. Nor can a growth inhibitor be postulated unless it is to be produced in liquid-layer cultures but not in shake flasks since the latter showed little effect of inoculum density upon growth rate.

In practice it is probably unnecessary to understand this effect so long as cultures are standardized at some inoculum between $10^{5}$ and ${ }_{10} 0^{6}$ spores $/ \mathrm{ml}$ chosen to give a combination 
of low 'auto-inhibition', high growth rate and high yield at a convenient time before the end of the exponential growth phase.

Valuable discussions and comparison of results with Dr A. J. Clutterbuck are gratefully acknowledged.

\section{REFERENCES}

BAINBRIDGE, B. W. (1971). Macromolecular composition and nuclear division during spore germination in Aspergillus nidulans. Journal of General Microbiology 66, 319-325.

ClutterbuCK, A. J. (1972). Absence of lactase from yellow-spored mutants of Aspergillus nidulans. Journal of General Microbiology 70, 423-435.

Pontecorvo, G., Roper, J. A., Hemmons, L. M., MacDonald, K. D. \& Bufton, A. W. J. (1953). The genetics of Aspergillus nidulans. Advances in Genetics 5, 141-238.

TrINCI, A. P. J. (1969). A kinetic study of the growth of Aspergillus nidulans and other fungi. Journal of General Microbiology 57, 1 I-24. 